\title{
Influence of propeller configuration on propulsion system efficiency of multi-rotor Unmanned Aerial Vehicles
}

\author{
B. Theys*, G. Dimitriadis, P. Hendrick, J. De Schutter \\ KU Leuven, Leuven, Belgium
}

\begin{abstract}
Multi-rotor Unmanned Aerial Vehicles make use of multiple propellers, mounted on arms, to produce the required lift. This article investigates the influence on propulsion system efficiency in hover due to the configuration of these propellers. Influence of pusher or puller configuration of the propeller, number of blades, shape and dimensions of the arm, coaxial and overlapping propellers, is presented. A dedicated test bench that allows testing of various experimental setups is designed and built in order to realistically represent multi-rotor arms. Test results show that a two-bladed pusher configuration is most efficient and slenderness of the arm has more influence on efficiency than shape. A coaxial propulsion system approaches the efficiency of a single-prop system at high disk loadings. Finally, interference effects due to overlapping propellers are discussed.
\end{abstract}

\section{INTRODUCTION}

Multi-rotor Unmanned Aerial Vehicles capable of Vertical Take-Off and Landing (VTOL UAVs), are increasingly deployed for various applications such as surveillance, autonomous parcel delivery, oil and gas spill detection and firefighting. Some of these applications demand long flight times which are often hard to achieve with the battery powered electrical propulsion system most multi-rotors use [1]. In order to extend the flight time, energy losses must be minimized. Energy losses occur in the battery due to internal resistance, the electronic speed controller (ESC) and the motor, as presented in [2]. Also the propeller and propulsion system configuration present losses due to interference with the multi-rotor arms and mutual interference between propellers. All these losses are schematically presented in figure 1 . While many papers focus on the blade geometry of the propeller [3] or propellerwing interaction losses in case of fixed-wing UAVs [4], this paper focusses on the effects of propeller configuration on the propulsion system efficiency of the multi-rotor. Five design choices that are often made during the design process of a multi-rotor are discussed in this paper, and investigated

*Email address: bart.theys@ @uleuven.be by a set of experiments. The first choice is between mounting the propellers downstream of the multi-rotor arms or upstream, often referred to as 'pusher' and 'puller' configuration respectively. A second design choice is the number of blades of the propeller; to this end a comparison between a two-blade and a three-blade propeller is presented. The influence of shape and thickness of the multi-rotor arm is discussed as a third design choice. Not much research has been published on this topic, however Fernandes [5] experimented with different arm shapes and found a small influence on the performance of the UAV. Finally, the mutual influence of propellers in coaxial and overlapping setups is presented. A similar study was performed by Nandakumar [6]: propellers were mounted with an overlap and a vertical offset, and different combinations were tested. In [7], a mathematical model and a computational-fluid-dynamics analysis is discussed for propeller wake interference on multi-rotor UAVs in high-speed forward flight.

\section{THEORY AND DEFINITIONS}

The propulsion system of a multi-rotor UAV consists of a battery, ESC, motor and propeller. Motor and ESC convert electrical power into mechanical power, delivered at the shaft of the motor. The efficiency of motor and ESC combined is therefore calculated as:

$$
\eta_{m o t+e s c}=\frac{\omega Q}{U I}=\eta_{m o t} \eta_{e s c}
$$

For the test setup in this paper, angular speed $\omega$ and torque $Q$ are measured at the motor shaft, while voltage $U$ and current $I$ are measured at the power source. The propeller uses the mechanical power to accelerate the air going through the disk area $A$ described by a rotating propeller. In hover, the efficiency of the propeller $\eta_{p l r}$ (also referred to as Figure of Merit in helicopter theory) is calculated using momentum theory as described by Rankine - Froude [8]:

$$
\begin{gathered}
V_{0}=0 \\
V_{1}=v_{i} \\
T=2 \rho A\left(v_{i}\right)^{2} \\
v_{i}=\sqrt{\frac{T}{2 \rho A}}
\end{gathered}
$$




$$
\begin{gathered}
P_{i}=T V_{1} \\
\eta_{p l r}=\frac{P_{i}}{P_{\text {mech }}}=\frac{T^{(3 / 2)}}{Q \omega \sqrt{2 \rho A}}
\end{gathered}
$$

Here, $V_{0}$ is the airspeed ahead of the propeller, $V_{1}$ is the air speed at the propeller disk and $V_{2}$ is the velocity behind the propeller disk after contraction of the flow tube as illustrated in figure 2. $T$ is the thrust produced by the propeller. $v_{i}$ is the induced velocity: the increase in velocity, induced by the propeller disk. The efficiency of the propeller $\eta_{p l r}$ is calculated as the ratio between the induced power $P_{i}$ and the required mechanical power $P_{\text {mech }} . \quad P_{i}$ is the theoretically required power to achieve the increase in air speed. A larger propeller diameter increases the disk area and decreases the required induced power. An innovative high-endurance multi-rotor using this principle is described by Verbeke [9]. The efficiency of the total propulsion system is calculated as:

$$
\eta_{\text {prop }}=\frac{P_{i}}{P_{\text {ele }}}=\frac{T^{(3 / 2)}}{I U \sqrt{2 \rho A}}=\eta_{p l r} \eta_{\text {mot }} \eta_{\text {esc }}
$$

Here, $U$ is the voltage of the power source and $I$ the delivered current to the propulsion system. In this paper the disk area $A$ is defined as the projected surface of the turning propeller. For a coaxial pair of propellers, this area is the same as for a single propeller of the same diameter.

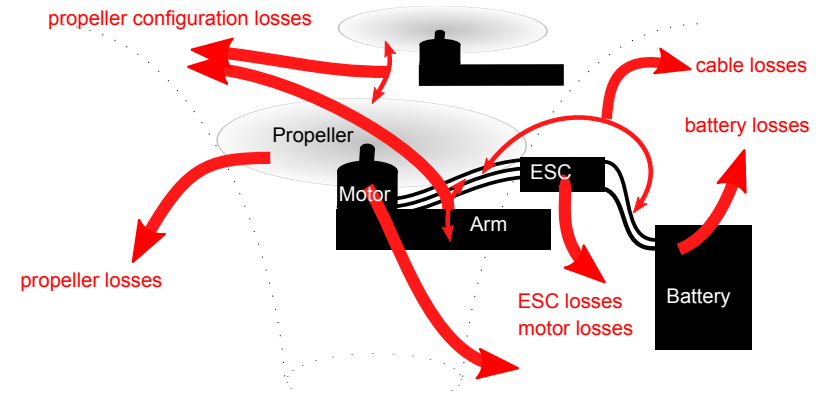

Fig. 1: Overview of losses from energy source to kinetic energy in the air.

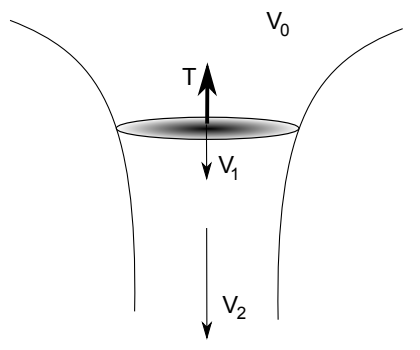

Fig. 2: Momentum theory applied on a propeller in hover condition.

\section{Experimental Setup}

Thrust and power, as well as rpm and torque are measured in order to calculate the efficiency of the motor with ESC and propeller separately using equations (1) and (8). Comparable test setups were described and used by Kotwani [10], Asson [11] and Hossain [12]. All these setups, however introduce a relatively large interaction between the propeller and the setup that is representative for a fuselage of a fixed-wing UAV but not for a multi-rotor arm. Figure 3 presents the experimental setup used in this paper. The setup is designed in a way that the addition of sensors introduces only a minimal change in shape compared to a typical multi-rotor arm. To this end, the torque of the motor is transmitted through a lever inside the arm and measured with force cell 1. This force cell is positioned at a lower point on the arm, out of the propeller slip stream and allows for measuring up to $1 \mathrm{Nm}$ with a resolution of $0.001 \mathrm{Nm}$. The arm with motor, propeller and torque sensor is mounted onto a pivot point. Thrust is measured by force cell 2 , at the other end of the pivot. Force cell 2 measures forces up to $50 \mathrm{~N}$ with a resolution of $0.01 N$. Rpm is measured by analysing the three-phase current between the ESC and the motor. The number of pulses per minute is divided by the number of poles of the DJI 2212/920KV motor in order to obtain the rpm. Voltage and current are measured at the DC power source, therefore power losses in the cable between the power source and ESC are incorporated in the efficiency measurement of the ESC+motor. The voltage of the power source is kept constant at $11.1 \mathrm{~V}$ throughout all experiments. This corresponds to the nominal voltage of a three cell lithium-polymer battery and is a suitable voltage for the combination of the motor with a Graupner 9x5 E-prop. The air density is taken into account, as calculated from a barometer, thermometer and humidity sensor measurement.

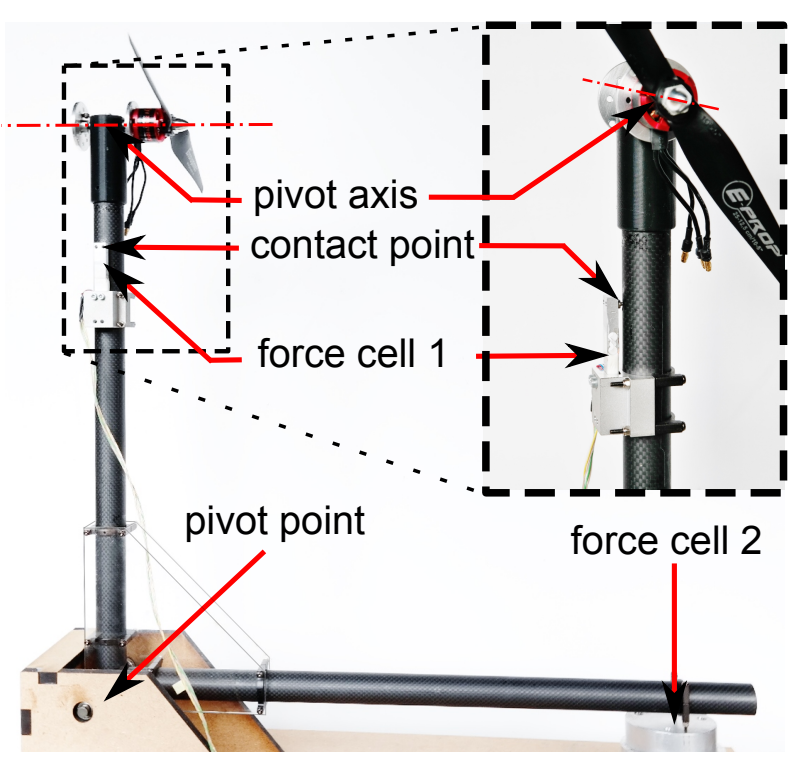

Fig. 3: Basic overview of the setup of the experiments. 


\section{EXPERIMENT}

A two-bladed Graupner 9x5 E-prop is used for all experiments described in this paper, except for the comparison with a three-bladed Graupner 9x5 E-prop. All experiments follow the same procedure: while holding the input voltage constant at $11.1 \mathrm{~V}$, the PWM (Pulse Width Modulated) signal to the ESC is increased in steps of $100 \mu s$ from $1200 \mu s$ up to $1900 \mu s$ and then again decreased by the same steps. This procedure gives two measurements per data point and averages out any hysteresis effects.

\subsection{Pusher vs.Puller}

The arm of the VTOL UAV supports the motor with propeller and can be mounted upstream or downstream of the propeller, respectively referred to as puller and pusher configuration. Both configurations are illustrated ${ }^{1}$ in figure 4 . This figure shows that the 'pusher' configuration requires a more complex landing gear in order not to obstruct the propellers. The efficiency of a two-blade and three-blade Graupner 9x5

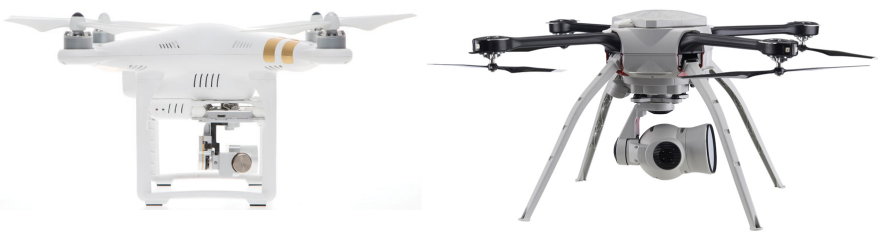

Fig. 4: Left: a multi-rotor with propellers in puller configuration. Right: a multi-rotor with propellers in pusher configuration.

propeller is measured up to full throttle for both pusher and puller configuration. Results are presented in figure 5. The left graph shows the propeller efficiency as a function of the disk loading of the propeller. The graph shows a strong increase of propeller efficiency that flattens out for higher disk loadings for all four configurations. A quadratic fit is made through the data points and presented as a solid line. For clear comparison, the relative difference with the two-blade puller configuration is plotted in the right graph of figure 5 . For both propellers, the pusher configuration results in an efficiency improvement of roughly $3 \%$ for disk loadings between $25 \mathrm{~N} / \mathrm{m}$ and $120 \mathrm{~N} / \mathrm{m}$.

\subsection{3-blade vs. 2-blade}

Most multi-rotors make use of propellers with two blades and these propellers are also most widely available. Some manufacturers also offer three-bladed versions of some of their propellers. In the following experiment a comparison is made between a two-blade and a three-blade Graupner 9x5 E prop. The data presented in figure 5, show that the threebladed propeller is less efficient with an average drop in efficiency of around $4 \%$. However, more blades result in a lower

\footnotetext{
${ }^{1}$ Source: http://www.dji.com/ and http://aeryon.com/.
}
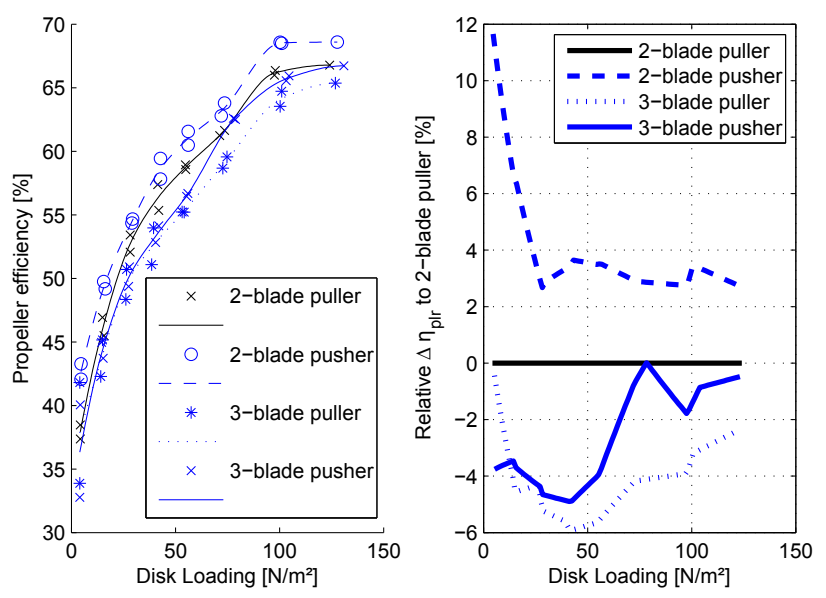

Fig. 5: Propeller efficiency comparison between pusher and puller configuration in combination with a two- or three-bladed propeller.

rpm for the same thrust, as presented by the graphs in figure 6 , and can therefore be interesting for reducing the noise.

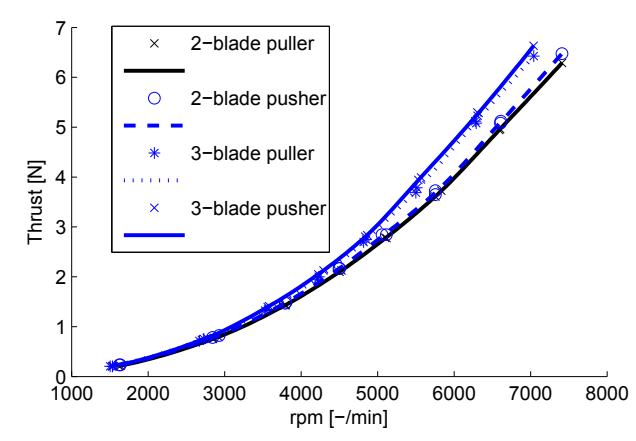

Fig. 6: Thrust as function of the rpm for a two-bladed and three-bladed propeller.

\subsection{Arm interaction}

For multi-rotors, a motor with propeller is mounted on an arm that is connected to the frame. This arm is subject to the airflow that the propeller induces and partially obstructs this flow, leading to a loss in thrust and therefore propulsion system efficiency. In the following experiment, three different types of arm are used in order to quantify their influence on the propulsion system efficiency. The first arm is a $25 \mathrm{~mm}$ cylindrical carbon tube, also used for the other experiments in this paper. The second arm is equipped with a $3 \mathrm{D}$ printed aerodynamically shaped nacelle that also incorporates the motor. The third arm is a thin $10 \mathrm{~mm}$ square carbon tube. The three arms are presented in figure 7 .

The left graph of figure 8 shows the propulsion system efficiency as a function of the disk loading for the three different 


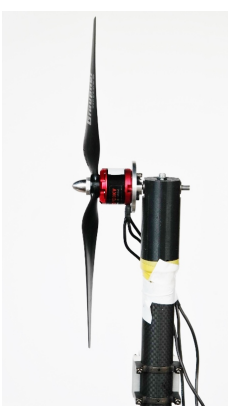

(a) $25 \mathrm{~mm}$ tube

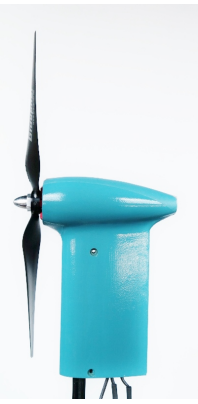

(b) nacelle

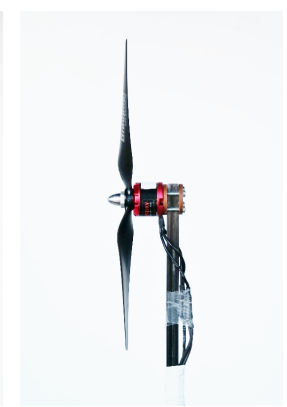

(c) $10 \mathrm{~mm}$ square tube
Fig. 7: Propeller and motor mounted to three different shapes of arm.

arms. Since in this experiment there is no space to include the torque sensor on the aerodynamically shaped and thin arm, it is not possible to measure propeller efficiency separately. The right graph of figure 8 shows the relative difference in propulsion system efficiency due to the different shape of arm. From these graphs, a noticeable efficiency increase is observed for the aerodynamically shaped arm and the thin arm. It is interesting to notice that the thin arm performs even better than the aerodynamically shaped arm, which was a somewhat unexpected result. Overall, the thin arm is clearly the best design choice, since in addition it is lighter than the other arms.
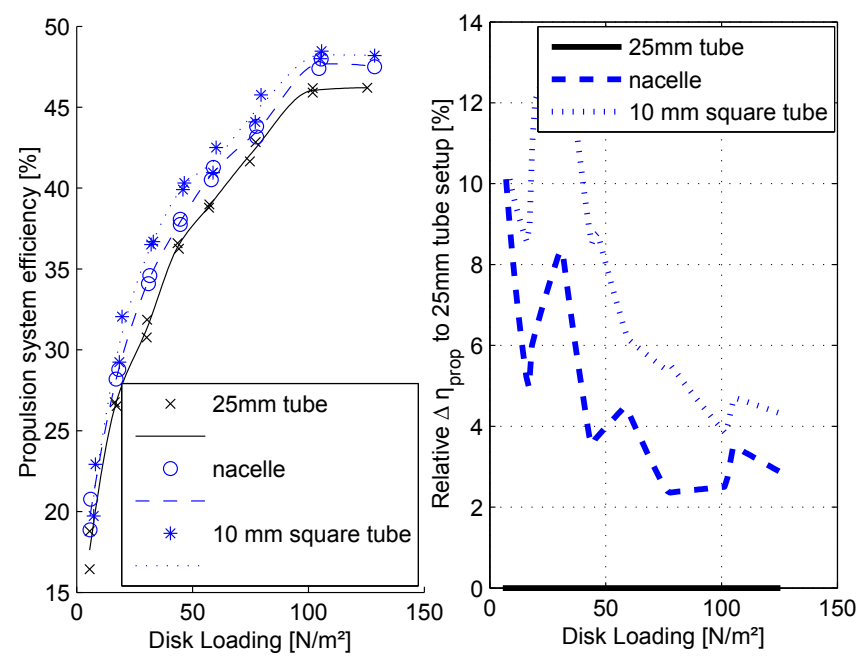

Fig. 8: Influence of arm dimensions and shape on propulsion system efficiency.

\subsection{Coaxial propellers setup}

It is often a topic of discussion whether a counter rotating pair of propellers, also referred to as coaxial propellers, is more efficient than a single propeller. Since coaxial propellers rotate in the opposite direction, swirl losses can be minimized compared to a single propeller. On the other hand, the downstream propeller operates in an air stream that is dis- turbed by the upstream propeller and can therefore lose efficiency. A carefully designed coaxial setup requires a downstream propeller with a slightly smaller diameter due to contraction of the flow, and a slightly higher pitch angle of the blades due to operation in an already accelerated flow. On most coaxial multi-rotors used nowadays however ${ }^{2}$, the upstream and downstream propellers are a counter rotating pair of identical propellers. Four different setups with coaxial Graupner 9x5 E propellers, as presented in figure 9, are tested. The performance resulting of each of these setups is presented in figure 10. The left graph does not reveal any large differences in propulsion system efficiency. However, from the right graph, showing the relative difference in propulsion system efficiency compared to the conventional coaxial setup, it becomes clear that a single propeller propulsion system is significantly more efficient than the coaxial setups, but the difference becomes smaller at higher disk loadings. At the highest disk loading of the single propeller at full throttle, the coaxial setups even become slightly more efficient. The right graph also shows that the conventional coaxial setup is more efficient compared to the other coaxial setups. There is no noticeable difference between $10 \mathrm{~cm}$ and $15 \mathrm{~cm}$ of spacing between the propellers, however for the setup in which the coaxial pair is very close at $3.5 \mathrm{~cm}$, there is a clear loss in efficiency. From these experiments, it is concluded that the propellers require some space in between and this space is best used to incorporate one arm, supporting both motors and propellers. It is also clear that coaxial propellers can be also interesting in terms of efficiency when high disk loadings are required for example if the UAV has to be compact or wind resistant. The additional weight due to the extra motor, ESC and propeller for the coaxial setup compared to a single propeller system, was not taken into account in the evaluation of the efficiency. In practice, this extra weight has to be subtracted from the produced thrust of the propulsion system since the system itself becomes heavier.

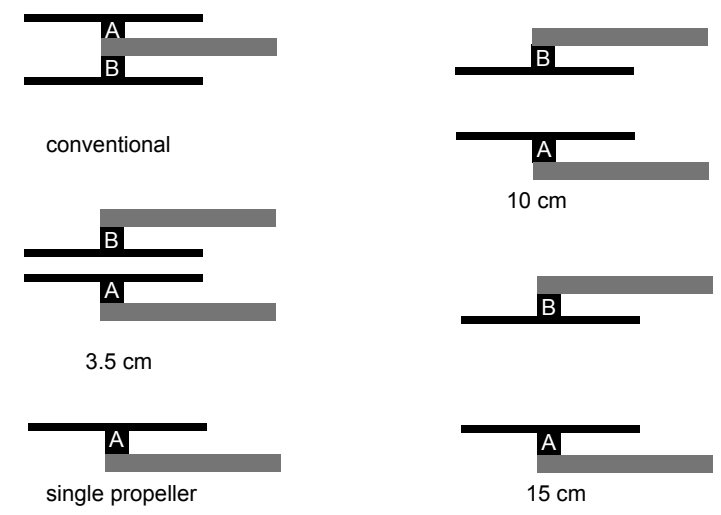

Fig. 9: Four different coaxial setups and a single propeller to compare with.

\footnotetext{
${ }^{2}$ For example: Aerialtronics Altura Zenith, Harwar Mega V8, 3DR X8+
} 

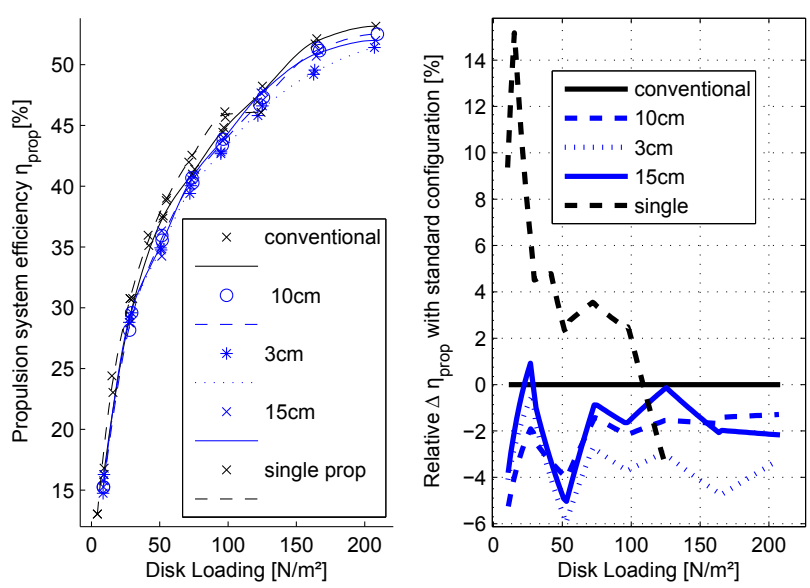

Fig. 10: Performance of different coaxial propeller setups.

\subsection{Partially overlapping propellers setup}

Larger propellers are able to deliver the same thrust for less power and are therefore preferred if long flight times are required. Since there is often a restriction on the dimensions of the UAV, as the diameter of the propellers increases, the tips of different propellers approach each other. In some designs propellers actually overlap [6]. The mutual influence of this overlap is tested in a series of experiments in which the axial distance and overlap of two propellers are varied. Figure 12 presents the used conventions of axial distance and overlap, in this paper presented as a percentage of the diameter of the propeller. Six different values for axial distance are used, being $[-100 \%,-50 \%,-$ $5 \%, 5 \%, 50 \%, 100 \%]$ and five different values of overlap are used, being $[-10 \%, 0 \%, 10 \%, 20 \%, 30 \%]$ of the diameter of the propeller. This results in a total of 30 configurations. Figure 11 shows the experimental setup. The marked area in this figure is used to calculate the efficiency of the double propulsion system with equation 8 . The area covered by the two overlapping propellers $A$ is calulated following relations:

$$
\begin{aligned}
\alpha & =2 \cos ^{-1}(1-\text { overlap }) \\
A & =\frac{1}{2}\left(\frac{D}{2}\right)^{2}(\alpha-\sin (\alpha))
\end{aligned}
$$

The conventions used for axial distance and overlap are presented schematically in figure 12. Propeller ' $A$ ' creates the disturbing flow for propeller ' $\mathrm{B}$ '. For a positive axial distance, propeller 'A' lies upstream of propeller ' $B$ ' and for a negative axial distance it lies downstream.

In this set of experiments, only the power and thrust of motor ' $\mathrm{B}$ ' is measured since for the set of experiments in which the axial distance is negative, motor ' $\mathrm{B}$ '. For every combination of distance and overlap, the power of motor ' $\mathrm{B}$ ' is measured for producing both $2.5 \mathrm{~N}$ and $5 \mathrm{~N}$ of thrust,

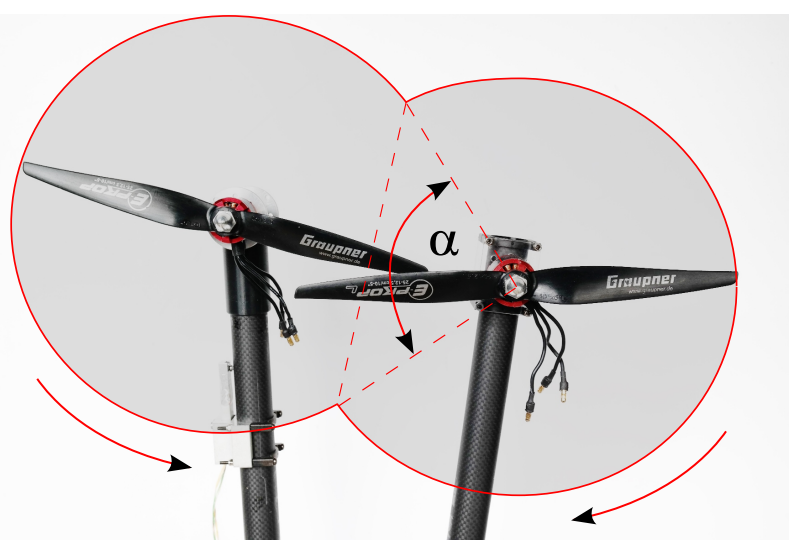

Fig. 11: Experimental setup that allows variation of axial distance and overlap between two counter rotating multi-rotor UAV propellers.

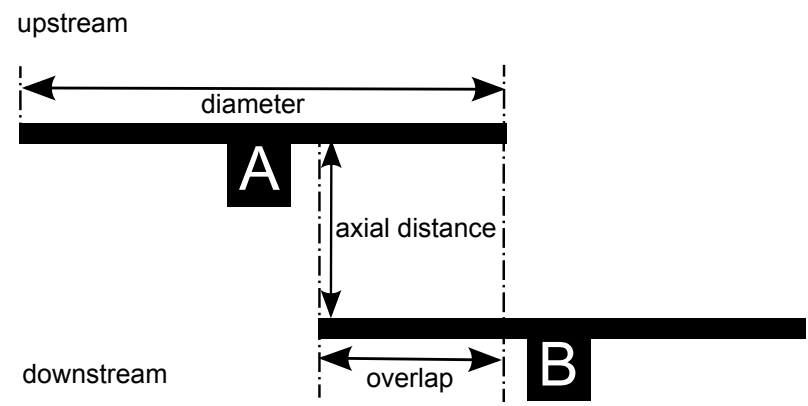

Fig. 12: Used conventions for the experiment with overlapping propellers.

which represents approximately $40 \%$ and $80 \%$ of the maximum thrust of the propeller in this setup. The relative difference in power compared to a single propeller without interaction of another propeller is presented in figure 13. Both graphs show an increase in required power for higher overlap. There is a large gradient between $-5 \%$ and $5 \%$ axial distance visible: the propeller requires less power when there is a propeller directly in front of it. This reduction is visible on both graphs and most pronounced for an overlap of $10 \%$. These results suggest that a propeller with a small overlap, located downstream of the other propeller at a small distance, has some benefit of this configuration since less power is required.

In order to evaluate the influence of propeller overlap on the propulsion system efficiency $\eta_{\text {prop }}$ two approaches are used.

- Overlapping propellers caused by increasing the propeller diameter:

For a multi-rotor with a fixed arm length, the diameter of the propellers can be increased in order to increase the disk area of the propellers $A$ and therefore 

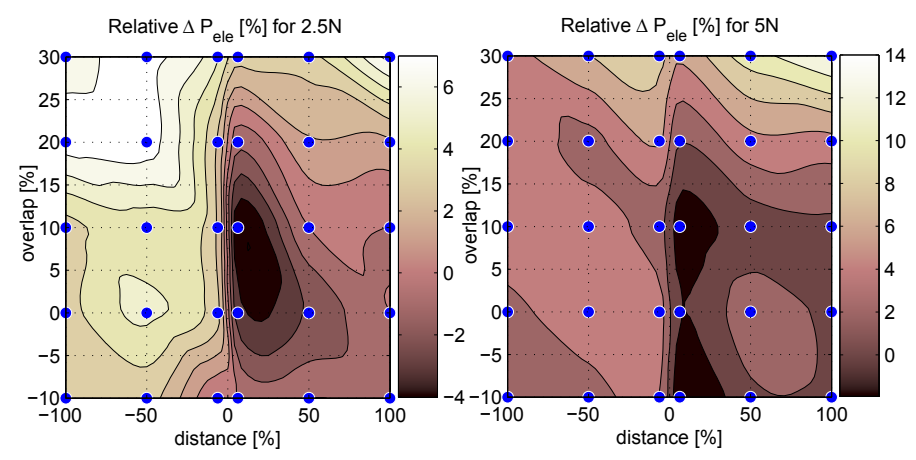

Fig. 13: Cubic interpolated contour plot of relative power increment for a single propeller in proximity to another propeller compared to a single propeller without interference, as a function of distance and overlap for $2.5 N$ (left) and $5 N$ (right) of thrust.

reduce the power required, resulting from equations 2 to 7 . In order to calculate the propulsion system efficiency with equation 8 , the combined disk area of the two propellers is calculated with equations 9 and 10. The relative difference in propulsion system efficiency compared to two propellers that are not in interaction is presented in figure 14. The left graph shows a clear decrease in efficiency for increasing axial distance between the propellers. From this graph, it can be seen that a slight overlap of $10 \%$ to $15 \%$ even increases the efficiency of a pair of counter rotating propellers when placed at a minimum axial distance. On the right graph, the same zone, $10 \%$ to $15 \%$ overlap and minimal axial distance, also shows the least decrease in efficiency. The highest decrease in efficiency is observed for high levels of overlap and increasing distance. Comparing the right graph the to left shows that the setup to produce $10 \mathrm{~N}$ of thrust has a higher overall efficiency decrease compared to the $5 N$ setup. From these graphs it can be concluded that the propulsion system efficiency of a pair of overlapping propellers decreases for higher loads and higher axial distance between the propellers. The best configuration for a pair of counter rotating and overlapping propellers is in a zone between $10 \%$ to $15 \%$ overlap at a minimal axial distance at low loads.

- Overlapping propellers caused by decreasing multirotor arm length:

For a multi-rotor with a fixed propeller diameter, the arm length can be decreased to make the UAV more compact and resulting in overlapping propellers. In order to evaluate the impact of this overlap on the propulsion system efficiency, the relative power increment is plotted on figure 15. These graphs show an increase in required power for all data points. The interesting zone between $10 \%$ to $15 \%$ overlap and minimal axial

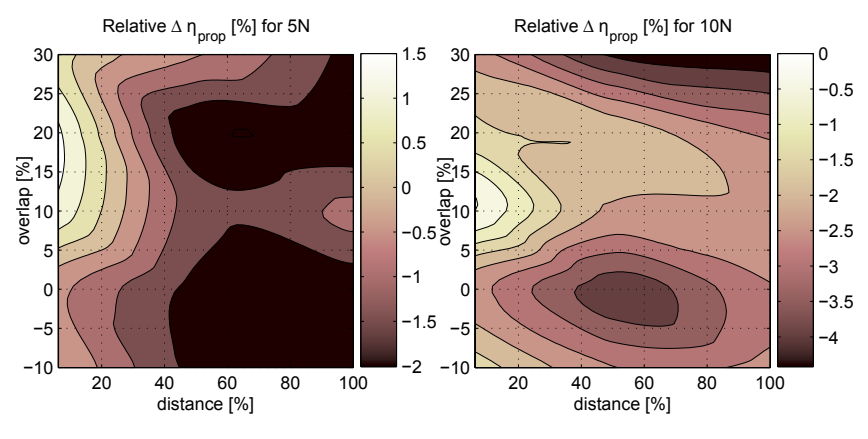

Fig. 14: Relative propulsion system efficiency increment (calculated based on the combined area of both overlapping propellers) for an overlapping pair of propellers compared to two single propellers without interference, as a function of distance and overlap for $5 \mathrm{~N}$ and $10 \mathrm{~N}$ of combined thrust.

distance shows to require not more energy than propellers that are non-overlapping but in close proximity to each other at $-10 \%$ overlap. For a compact multirotor design, letting the propellers overlap with $10 \%$ while keeping the axial distance minimal is the best design choice.
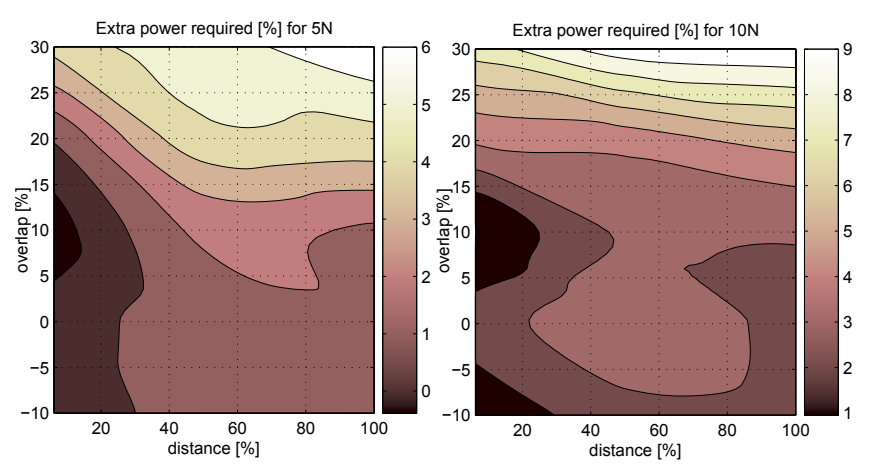

Fig. 15: Relative required power increment for an overlapping pair of propellers compared to two single propellers without interference, as a function of distance and overlap for $5 \mathrm{~N}$ and $10 \mathrm{~N}$ of combined thrust.

\section{Conclusion}

This paper discusses the effects of propeller configuration on the propulsion system efficiency of a multi-rotor. Five design choices are studied. A pusher configuration proves to be preferable in terms of efficiency in hover conditions. An increase of 2 to $4 \%$ in efficiency is measured. This increase is small, however, and requires a taller more complicated integration of the landing gear, resulting in more weight. A three-bladed variant of the tested propeller results in a lower efficiency in the order of 2 to $6 \%$ but can be beneficial to reduce noise and risk due to its lower required rpm. 
Tests with three different arms on which the propulsion system is mounted, show that a thin rectangular arm is more efficient compared to a slightly thicker but aerodynamically shaped arm and can improve efficiency compared to a thick arm with 4 to $8 \%$. The difference between the three arms becomes less pronounced for higher disk loadings. At low disk loadings, the propulsion system setup with a coaxial set of propellers is less efficient compared to the setup with a single propeller. However this difference becomes smaller at higher disk loadings at which a single propeller propulsion system almost reaches its maximum thrust. For a pair of overlapping propellers, power demand increases with the percentage of overlap. The best configuration for overlapping propellers was observed to be in a zone between $10 \%$ to $15 \%$ overlap while keeping minimal axial distance. This configuration allows to use larger propellers or decrease the length of the multi-rotor arms without decreasing the propulsion system efficiency. The overlapping configuration is also more interesting for propellers with a low disk loading.

\section{ACKNOWLEDGEMENTS}

A special thanks to Menno Hochstenbach, who implemented the rpm measurement based on the three-phase current between motor and ESC and Jon Verbeke for the fruitful discussions on the test results.

\section{REFERENCES}

[1] Mauro Gatti, Fabrizio Giulietti, and Matteo Turci. Maximum endurance for battery-powered rotary-wing aircraft. Aerospace Science and Technology, 2015.

[2] Grzegorz Szafranski, Roman Czyba, and Marian Blachuta. Modeling and identification of electric propulsion system for multirotor unmanned aerial vehicle design. In Unmanned Aircraft Systems (ICUAS), 2014 International Conference on, pages 470-476. IEEE, 2014.

[3] Moses Bangura, Marco Melega, Roberto Naldi, and Robert Mahony. Aerodynamics of rotor blades for quadrotors. arXiv preprint arXiv:1601.00733, 2016.

[4] Sergey Shkarayev, J Moschetta, and Boris Bataille. Aerodynamic design of vtol micro air vehicles. In Proc. of the MAV07 International Conference, France. Citeseer, 2007.

[5] Nelson Fernandes. Design and construction of a multirotor with various degrees of freedom. $\mathrm{PhD}$ thesis, MS Thesis, Technical Univ. of Lisboa, 2011.

[6] Ganeshram Nandakumar, Thiyagarajan Ranganathan, BJ Arjun, and Asokan Thondiyath. Design and analysis of a novel quadrotor system-voops. In Robotics and Automation (ICRA), 2015 IEEE International Conference on, pages 1692-1697. IEEE, 2015.
[7] Jinglin Luo, Longfei Zhu, and Guirong Yan. Novel quadrotor forward-flight model based on wake interference. AIAA Journal, 53(12):3522-3533, 2015.

[8] W Froude. On the elementary relation between pitch, slip, and propulsive efficiency. 1920.

[9] Jon Verbeke, Dries Hulens, Herman Ramon, Toon Goedeme, and Joris De Schutter. The design and construction of a high endurance hexacopter suited for narrow corridors. In Unmanned Aircraft Systems (ICUAS), 2014 International Conference on, pages 543-551. IEEE, 2014.

[10] Kailash Kotwani, SK Sane, Hemendra Arya, and K Sudhakar. Experimental characterization of propulsion system for mini aerial vehicle. In 31st National Conference on FMFP, pages 16-18, 2004.

[11] Kenneth M Asson and Patrick F Dunn. Compact dynamometer system that can accurately determine propeller performance. Journal of Aircraft, 29(1):8-9, 1992.

[12] Mohammed R Hossain and Nicholas Krouglicof. Propeller dynamometer for small unmanned aerial vehicle. In Electrical and Computer Engineering (CCECE), 2010 23rd Canadian Conference on, pages 1-5. IEEE, 2010. 